\title{
What is the quality of smoking cessation advice in guidelines of tobacco-related diseases?
}

\author{
Authors: Ilze Bogdanovica, ${ }^{A}$ Sanjay Agrawal, ${ }^{B}$ Benjamin Gregory, ${ }^{C}$ John Britton ${ }^{D}$ and Jo Leonardi-Bee ${ }^{E}$ on behalf \\ of the Tobacco Advisory Group of the Royal College of Physicians
}

Smoking is a major risk factor for a range of diseases, and quitting smoking provides considerable benefits to health. It therefore follows that clinical guidelines on disease management, particularly for diseases caused by smoking, should include smoking cessation. The aim of this study was to determine the extent to which this is the case. We conducted a systematic review investigating clinical guidelines and recommendations issued by UK national or European transnational medical speciality associations and societies issued between 2000 and 2012 on a range of diseases caused by smoking. We then investigated whether selected guidelines contained reference to smoking cessation and smoking cessation advice. Although the extent to which smoking and smoking cessation was mentioned in the guidelines varied between diseases, only $60 \%$ of guidelines identified recognised that smoking is a risk factor for the development of the disease and $40 \%$ recommended smoking cessation. Only $19 \%$ of guidelines provided detailed information on how to deliver smoking cessation support. Smoking cessation is not comprehensively addressed in current UK and transnational European clinical practice guidelines and recommendations.

KEYWORDS: Smoking, smoking cessation, guidelines, systematic review

\section{Introduction}

Tobacco use is the biggest cause of preventable morbidity and mortality in the UK. ${ }^{1}$ The diseases it causes or exacerbates

Authors: ${ }^{A}$ research fellow, Centre for Tobacco and Alcohol Studies, University of Nottingham, Nottingham, UK; ${ }^{B}$ consultant in respiratory and intensive care medicine, Department of Infection, Immunity and Inflammation, Institute for Lung Health, Department of Respiratory Medicine, Glenfield Hospital,

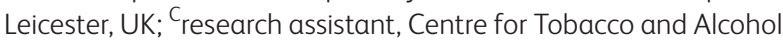
Studies, University of Nottingham, Nottingham, UK; D professor of epidemiology and director, Centre for Tobacco and Alcohol Studies, University of Nottingham, Nottingham, UK; Eprofessor, Centre for Tobacco and Alcohol Studies, University of Nottingham, Nottingham, UK include cancer, heart disease, lung disease, stroke, peripheral vascular disease, diabetes, infection, dementia and macular degeneration. ${ }^{2}$ In addition to premature mortality, these diseases cause disability that contributes to social and economic disadvantage, ${ }^{3}$ loss of productivity, ${ }^{4}$ and dependence on family or socially funded carers. The financial cost to society of these and other effects of tobacco smoking is estimated to be in excess of $£ 13$ billion per annum. ${ }^{5}$

This individual and societal burden of disease can be prevented for future generations by preventing the uptake of smoking among young people. ${ }^{6}$ However, reducing this burden in the more immediate future is dependent on helping as many current smokers as possible to quit smoking. This is particularly relevant to smokers who have already developed a disease caused or exacerbated by smoking, since stopping smoking can arrest or in some cases reverse the progression of their disease. ${ }^{7}$ It therefore follows that smoking cessation interventions or reference to published smoking cessation guidelines should be a key component of the clinical management of these diseases, and hence also of published guidelines or their updates on their clinical management.

We have therefore systematically examined clinical management guidelines produced by professional societies and governmental agencies in the UK and Europe for a range of smoking-related diseases, to assess the extent to which smoking cessation interventions are prioritised within them.

\section{Methods}

We carried out a series of systematic reviews of clinical guidelines and clinical recommendations published since 2000 and produced under the auspices of a relevant UK national or European transnational medical speciality association, professional society or government agency, and relating to a range of diseases caused or exacerbated by smoking comprising cancer of the lip, oral cavity and pharynx, oesophagus, lung, stomach, pancreas, kidney, pelvis or bladder; cardiovascular disease (including ischaemic heart disease, angina, myocardial infarction and aortic aneurysms); cerebrovascular disease (including stroke, transient ischaemic attack and brain aneurysm); or respiratory disease (pneumonia and chronic obstructive pulmonary disease (COPD)). We excluded guidelines produced by and for individual European countries outside the UK. 
A series of search strategies were developed for two electronic databases (Medline and EMBASE) using recognised search filters for identifying clinical guidelines as developed by the Guideline Resource Centre ${ }^{8}$ for each disease. Disease-specific terms were developed using the relevant search terms as developed by Cochrane Review Groups. Searches were typically conducted to the end of 2013. We additionally searched the National Library for Health Guidelines Finder, the TRIP Database and the International Guideline Library.

We then searched individual guideline documents to establish whether i) smoking was identified as a risk factor or major cause of disease; ii) smoking cessation interventions were recommended in disease management; and iii) whether there was reference or a link to specific smoking cessation guidelines (for example the National Institute for Health and Care Excellence guidelines for various diseases provide reference to smoking cessation guidelines rather than include specific treatment recommendations) or recommendations on evidence-based treatments for smoking cessation, including behavioural support and pharmacotherapy, or referral to a stop smoking service.

The titles, abstracts, and full texts were screened by two reviewers independently to select eligible guidelines using previously piloted checklist forms. Disagreements were resolved through discussion with a third reviewer. One reviewer extracted data relating to the agency responsible for developing/ issuing guidelines, country of origin, year of publication, status of clinical guidelines (new or updated), and level of development (UK/European) using a piloted data extraction form. Simple descriptive summary statistics are used to report the presence of the smoking cessation advice within the guidelines; summaries of these studies are also reported.

\section{Results}

Our searches identified 141 eligible clinical guidelines, of which 37 were produced in the UK and 104 by a European medical speciality association, relevant professional society or government agency. Two of the guidelines were included more than once as they had distinct sections on more than one eligible cancer under investigation, ${ }^{9,10}$ thus a total of 144 guidelines were included in the review. Of the 144, 86 (60\%) referred to smoking as a risk factor for the development of the disease; 57 (40\%) provided a statement regarding smoking cessation; and 28 (19\%) made reference to specific treatments for smoking cessation or to a smoking cessation guideline (Table 1). A full list of eligible guidelines is provided in Table S1 (available online) and cessation content by disease broken down as follows:

\section{Cancers}

Lip, oral cavity and pharynx cancer

From 4,452 titles identified in the searches, 46 were selected based on title and abstract. Following full-text screening, two guidelines, both from the UK, were included. ${ }^{11,12}$ Both mentioned smoking as a risk factor for head and neck cancer and highlighted the importance of smoking cessation, and provided details of specific treatments or else referred to a guideline for smoking cessation.

\section{Oesophageal cancer}

From 1,884 titles identified, 22 were selected based on title and abstract, and 8 included after full-text screening. 9,10,13-18 In total, 4 guidelines were from the $\mathrm{UK}^{9,10,13,15}$ and 4 were European. ${ }^{14,16-18}$ Only 4 guidelines (50\%), 3 UK and 1 European, mentioned smoking as a risk factor for oesophageal cancer. All 3 of these UK guidelines discussed the benefits of smoking cessation, ${ }^{9,13,15}$ but only 1 provided a link to smoking cessation management guidelines. ${ }^{9}$

Lung cancer

From 4,825 titles identified from the searches, 75 were selected on the basis of titles and abstracts and 26 following full-text screening, ${ }^{19-44} 1$ of which ${ }^{38}$ was a summary of more

Table 1. Summary of clinical guidelines and recommendations with the presence of smoking and smoking cessation.

Guidelines referring to smoking, $n$ (\%)

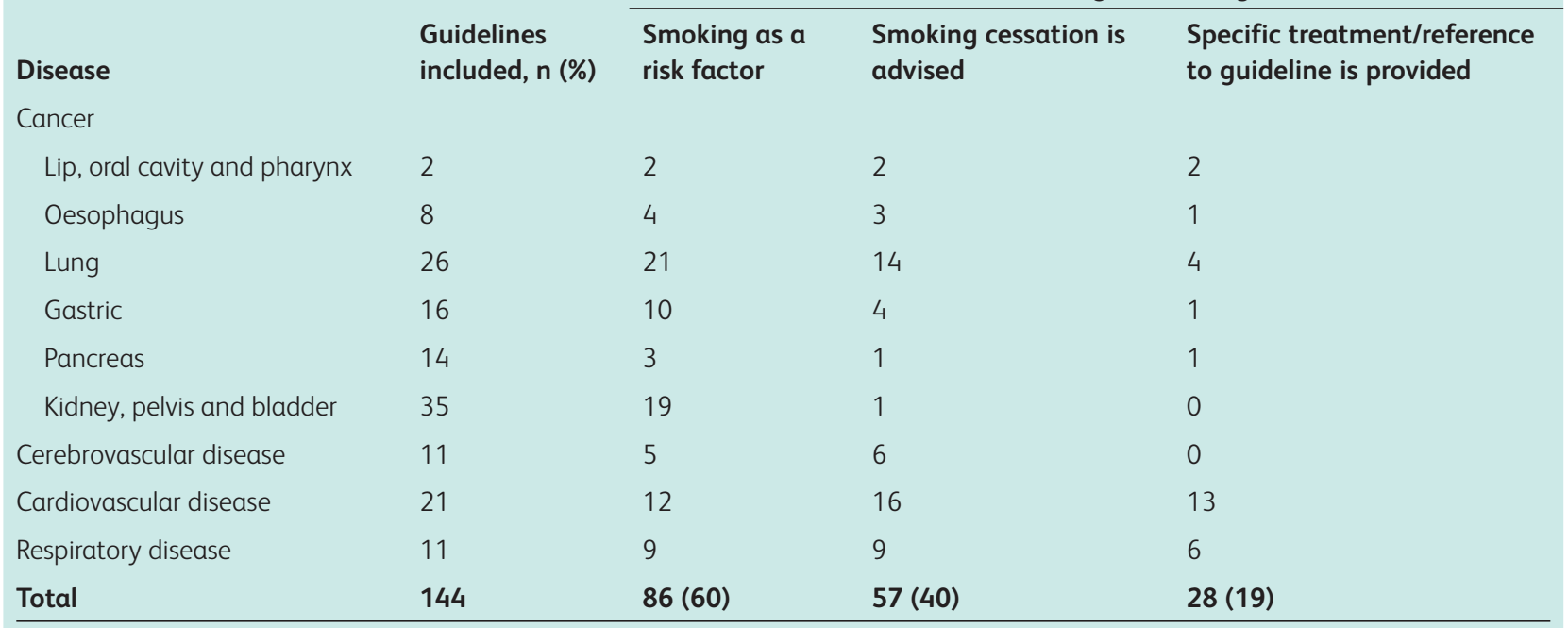

Two of the guidelines included more than one cancer under investigation. ${ }^{9,10}$ 
extensive guidance published by the National Institute for Health and Care Excellence (NICE). ${ }^{22,23,37,43} 21$ (81\%) of these 26 guidelines made reference to the role of smoking as a cause of lung cancer; ${ }^{19,20,21-32,34,35,37,40,42-44} 14$ (54\%) referred to smoking cessation, , $^{21-23,26,30,32-35,37,38,40,43,44}$ and 4 $(15 \%)$ provided specific advice or reference to guidelines on smoking cessation. ${ }^{22,23,37,43}$ Of these 4,3 were $\mathrm{UK}^{22,23,37}$ and 1 European-level ${ }^{43}$ guidelines.

Gastric cancer

From 7,259 titles identified from the searches, 34 were selected based on title and abstract and 16 after fulltext screening., ${ }^{9,45-58} 10$ of the included guidelines $(63 \%)$ referred to smoking as a risk factor for gastric cancer ${ }^{9,10,45-47,49,51,53,55,57}$ but only 4 (25\%; all UKbased) recommended that smoking cessation should be advised, ${ }^{9,10,45,47}$ and only 1 of these provided a link to smoking cessation management guidelines. ${ }^{9}$

\section{Pancreatic cancer}

From 1,636 titles identified from the searches, 29 were selected on title and abstract and 14 after full-text screening., ${ }^{99-71}$ Only $3\left(21 \% ; 2 \mathrm{UK}^{9,59}\right.$ and 1 European $\left.^{71}\right)$ of these 14 guidelines referred to smoking as a risk factor for pancreatic cancer, and 1 referred to smoking cessation and provided a link to smoking cessation management guidelines.

\section{Kidney, pelvis and bladder cancer}

From 4,411 titles identified from the searches, 67 were selected based on titles and abstracts and 35 after full-text screening. ${ }^{72-106} 19$ (54\%) of these reported that smoking increased the risk of disease, ${ }^{72,75,76,80,81,83,86-88,91,93,95-102}$ but only 1 referred to smoking cessation ${ }^{76}$ and none included any link or recommendation on smoking cessation therapy.

\section{Cerebrovascular disease}

From 12,414 titles identified, 78 were potentially eligible based on title and abstract and 11 after full-text screening. ${ }^{107-117}$ $5(36 \%)$ of these mentioned smoking as a risk factor for cerebrovascular disease; ${ }^{110,111,116} 6$ (55\%; 3 UK and 3 European) recommended smoking cessation; ${ }^{110,111,115,116}$ and none referred to specific treatments or referenced any smoking cessation guidelines.

\section{Cardiovascular disease}

From 13,351 titles identified in the searches, 135

were selected based on titles and abstracts and

21 after full-text screening. ${ }^{118-138}$ Of these 21,13

$(62 \%)$ reported that smoking increased the risk of disease; ${ }^{118-120,122-124,126,127,131,132,136,138} 17$ (81\%) recommended smoking cessation; ${ }^{118,120-123,125-128,130-132,134,136-138}$ and 13 (62\%; 7 UK and 6 European) made reference to specific treatments or smoking cessation guidelines. ${ }^{118,120,122,125-127,129,132,133,135-138}$

\section{Respiratory disease}

From 13,730 hits identified from the searches, 103 were selected based on title and abstract and 11 after full-text screening. ${ }^{139-149}$ $9(82 \%)$ of these mentioned smoking as a risk factor for COPD or pneumonia; ${ }^{139-143,145-149} ; 9(82 \%)$ referred to smoking cessation; ${ }^{139-143,145-148}$ and 6 (55\%; 3 UK and 3 European) referred to specific treatments or referenced smoking cessation guidelines. $^{140-142,145,146,148}$

\section{Discussion}

Tobacco-related diseases, and specifically cardiovascular disease, cancer and COPD, account for $80 \%$ of deaths in Europe. ${ }^{150}$ Smoking cessation should be a priority in the management of all such conditions, and promoted in management guidelines. However, the present paper, which is to our knowledge the first systematic review of this, found that $40 \%$ of the guidelines we identified did not identify smoking as a risk factor for the disease; only $40 \%$ recommended smoking cessation in the management of the disease; and only $19 \%$ provided information on specific smoking cessation treatments or else referred to definitive smoking cessation guidance. These findings varied substantially between disease categories however, with over half of guidelines on respiratory and cardiovascular disease providing smoking cessation guidance, but with much lower proportions among cerebrovascular disease and cancer guidelines. UK guidelines were generally more likely to include smoking cessation guidance than European guidelines, with the majority citing smoking cessation guidance from the NICE. ${ }^{151}$ We acknowledge that not all clinical guidelines for specific diseases could include full smoking cessation guidelines, but would expect guidelines on diseases caused by smoking to acknowledge and advise on the need to quit smoking. We therefore defined reference to existing smoking cessation guidelines where further information could be found as sufficient.

Smoking cessation increases both quantity and quality of life expectancy at almost any age. In respiratory disease, smoking cessation reduces the rate of decline in lung function among patients with COPD; ${ }^{152}$ and reduces the risk of lung infections and of asthma exacerbation. ${ }^{153}$ In lung cancer, smoking cessation at diagnosis in early stage disease is associated with significantly improved survival. ${ }^{154}$ In cardiovascular disease, smoking cessation reduces the risk of recurrence by around $50 \%$ over the longer term, and in cerebrovascular disease by around 15\%. ${ }^{155-159}$ Smoking cessation should therefore be a high priority in managing these diseases, and it would therefore be reasonable to expect definitive management guidelines to recognise this. Our finding that even in respiratory and cardiovascular disease, reference to appropriate smoking cessation guidance or provision of specific advice was far from universal indicates that smoking cessation is not receiving the attention it merits in the management of these conditions.

For the less common cancers caused by smoking, such as cancer of the oesophagus, pancreas and bladder, inclusion of cessation guidance was generally (with the exception of guidance on cancer of the lip, oral cavity or pharynx) even less common. There is evidence that smoking cessation improves life expectancy in early stage lung cancer, cardiovascular and respiratory disease $;{ }^{153,154}$ and smoking cessation is likely to have a significant impact in also preventing intercurrent infections, ${ }^{160,161}$ promoting wound healing, ${ }^{162-164}$ and other benefits to the management of these conditions. It is therefore surprising that smoking cessation is afforded so little priority in the management of these less common cancers. The impact of this omission is likely to be inadequate treatment of tobacco addiction, with the consequence of an excess of several hundred thousand preventable deaths and significant morbidity.

Why is this omission so common? There are many potential plausible causes. The authors of these guidelines may be 
experts in specific disease but may not be so knowledgeable in understanding the links between tobacco use and disease progression or the benefits of smoking cessation in established disease. ${ }^{150,154,165-167}$ Authors may also underestimate the strength of tobacco addiction, ${ }^{168}$ the difficulty overcoming this addiction ${ }^{169}$ or may lack the knowledge of the most effective methods to achieve smoking cessation. ${ }^{170}$ In addition, authors may be influenced by other barriers that effect health professionals discussing smoking cessation with patients such as time, knowledge, perceived lack of efficacy or their own use of tobacco. ${ }^{171-173}$ A less benign reason for our findings may be the influence of device manufacturers and pharmaceutical companies on guideline production, ${ }^{174}$ since disease prevention runs contrary to their commercial interest. Finally, it may be that authors of guidelines are focusing on novel procedures or pharmacotherapy and weight these recommendations higher than more 'conservative' measures despite the effectiveness of the latter. ${ }^{175}$

We used robust search strategies and screening methods to identify and assess the eligibility of the clinical guidelines included in the review; therefore it is unlikely that any guidelines we did not identify would have a significant impact on our findings. The indexing of clinical guidelines is still being developed; therefore our search strategies were very sensitive and identified many articles that were not relevant. Some of the included guidelines were in the form of a very short consensus document or a shortened updated guideline; therefore, due to the restrictions on the length of the documents it is likely that smoking or smoking cessation were not mentioned. However, if the disease is caused by smoking or smoking contributes to its development and smoking cessation could reduce risk of the disease or death, it should be mentioned in the short versions or updates of guidelines, for example, by providing a reference to existing guidelines as often observed in NICE guidelines. Our finding that major guidelines on cardiovascular disease, cancer and respiratory diseases across Europe neglect smoking prevention is likely to reflect low levels of intervention to prevent smoking among people with smoking-related disease, and a consequent failure to avoid significant morbidity and mortality.

\section{Conclusion}

A plethora of guidelines is produced each year by a variety of institutions and professional societies to disseminate research findings, best practice and to reduce variation in care. Their quality is variable, and dissemination and impact of guidelines often poor. ${ }^{176}$ We hope that by drawing attention to this deficiency in the inclusion of smoking cessation we can improve this, and that this will be reflected in changes in clinical practice. For the general standard of smoking cessation advice and intervention to be raised, government and peer review of practice standards, government health policy driving change, smoke-free legislation, commissioning of services and anti-tobacco advocacy groups will be needed.

\section{Funding}

Funded by the University of Nottingham.

\section{References}

The full reference list can be accessed online at www.clinmed. rcpjournal.org/content/current

Address for correspondence: Dr I Bogdanovica, Centre for Tobacco and Alcohol Studies, University of Nottingham, City hospital campus, Nottingham NG5 1PB, UK.

Email: ilze.bogdanovica@nottingham.ac.uk

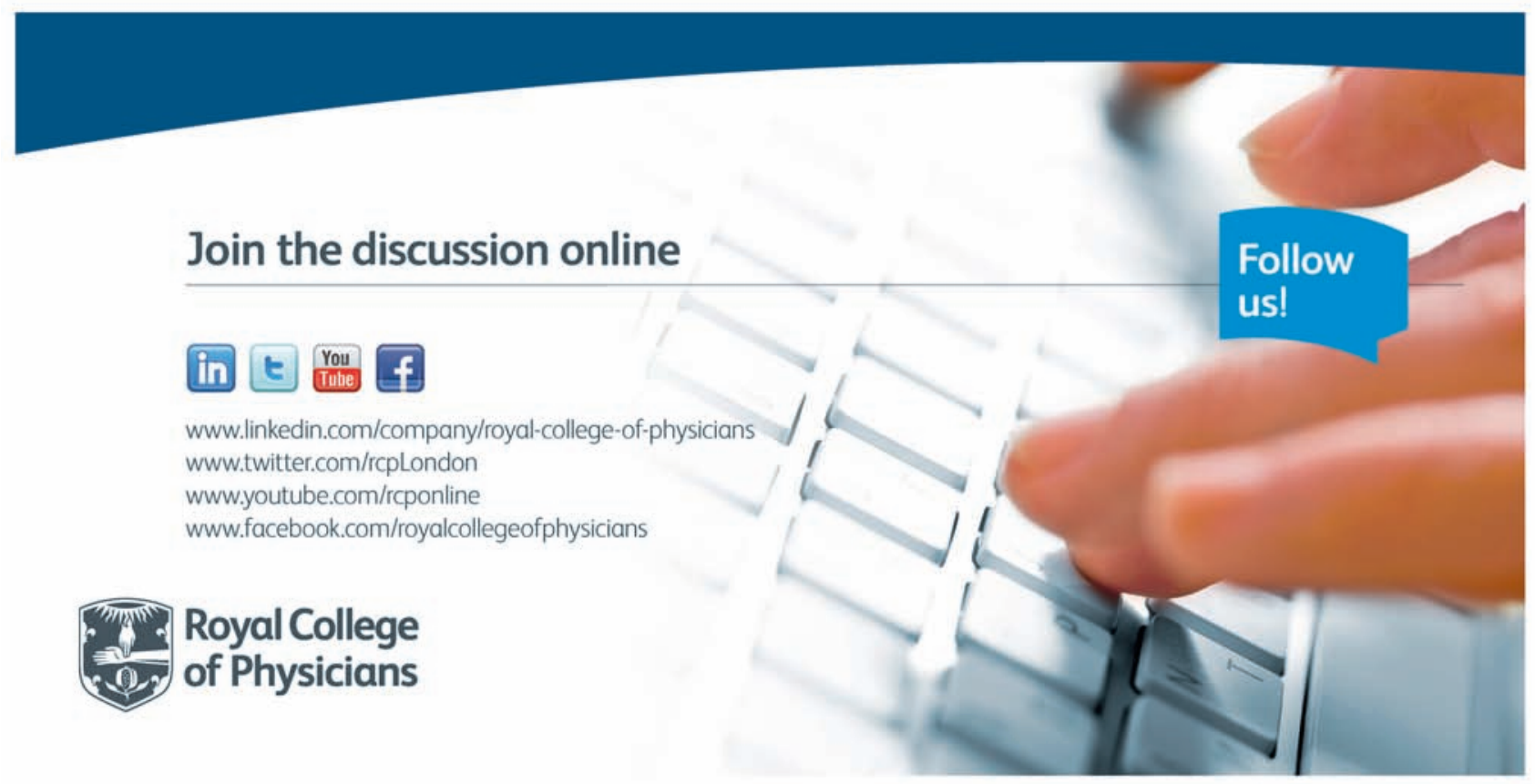

